\title{
Association between mental health and nutritional status in university students aged 18-24 years
}

\section{Abstract}

It is estimated that $19 \%$ of $16-24$ year olds suffer from a mental health condition. There has been a fivefold increase in reported mental health problems from first year university students since $2006 / 07^{(1)}$. Limited but consistent evidence suggests that nutritional status is linked to mental health. University students may be more vulnerable to the impact of diet on mental health due to increased food insecurity and risk of stress. The aim of this project was to determine the association between nutritional status and stress, anxiety and depression in university students.

After obtaining ethical approval, university students $(n=562)$ aged $18-24$ y were recruited. A cross-sectional design was used to measure body mass index $(\mathrm{BMI})$, percent body fat $(\% \mathrm{BF})$, capillary blood measures of glucose and lipids and nutrient intake by validated 3-day food diary. Mental health status was determined by DASS21 questionnaire ${ }^{(2)}$ and participants were grouped into 5 categories: normal, mild, moderate, severe and extremely severe for anxiety, depression or stress. Difference in nutritional status between categories was determined by a Kruskal-Wallis test and Dun-Bonferroni post-hoc tests using SPSS version 25 and significance determined as $p<0.05$.

Results showed that depression was associated with $\%$ BF $(p=0.025)$, blood glucose $(p<0.001)$, dietary protein $(p=0.003)$ and dietary sugar $(p=0.041)$. Anxiety was associated with blood glucose $(p<0.001)$ and dietary protein $(p=0.007)$.Stress was associated with total cholesterol $(p=0.007)$, HDL cholesterol $(p=0.007)$ and blood glucose $(p<0.001)$.

In light of these findings, further research is required to investigate the effect of student lifestyle habits and university practices on mental health and nutritional status so that strategic interventions can be designed to improve the health of young adults at university.

\section{Conflict of Interest}

There is no conflict of interest

\section{References}

1. Institute for Public Policy Research (2017) Not by degrees: improving student mental health in the UK's universities. Available at: https://www.ippr. org/research/publications/not-by-degrees [Accessed on 05/04/19].

2. Lovibond SH \& Lovibond PF (1995). Manual for the Depression Anxiety Stress Scales. (2nd. Ed.) Sydney: Psychology Foundation. 